\title{
Status and Recent Results of the ATLAS Experiment
}

\author{
Alexander Cheplakov ${ }^{1}$, on behalf of the ATLAS Collaboration \\ Laboratory of High Energy Physics, JINR \\ 141980 Dubna, Russia \\ E-mail: Alexander. Cheplakovecern.ch
}

The Large Hadron Collider at CERN is continuing its operation in 2012 showing a stable performance at $4 \mathrm{TeV}$ per proton beam energy and successfully providing enough statistics for the Higgs boson discovery. ATLAS is one of four major experiments taking data since the LHC start-up. The status of the detector and its performance are presented together with highlights of main results, including the observation of a Higgs-like particle, some studies of Standard Model measurements, and some heavy ion results. The ATLAS upgrade plans are presented.

XXI International Baldin Seminar on High Energy Physics Problems

JINR, Dubna, Russia

September 10-15, 2012

1

Speaker 


\section{ATLAS Detector}

ATLAS is a general-purpose detector designed to explore the full discovery potential of the Large Hadron Collider (LHC), including searches for the Higgs boson, SUSY particles and new heavy gauge bosons $(W, Z)$. The detector (Fig. 1) consists of two cylindrical electromagnetic spectrometers, electromagnetic and hadronic calorimeters with a pseudorapidity coverage up to 4.9. The vertices and tracks of charged particles are reconstructed in the inner detector consisting of silicon pixel, silicon strip and transition radiation detectors located in a 2 $\mathrm{T}$ magnetic field provided by a superconducting solenoid. The tracking detector covers the pseudorapidity range \pm 2.5 . It is surrounded by a calorimeter system. Highly granular liquid argon (LAr) electromagnetic sampling calorimetry together with LAr hadronic endcaps and Fescintillating tiles provide an excellent performance for reconstruction of electromagnetic and hadronic showers. The muon spectrometer is positioned outside the calorimeters. The air-core toroid system has a strong bending power with an average magnetic field integral of about 3 Tm. The drift tubes and cathode strip chambers provide precise coordinate measurements while the resistive-plate chambers are used for triggering.

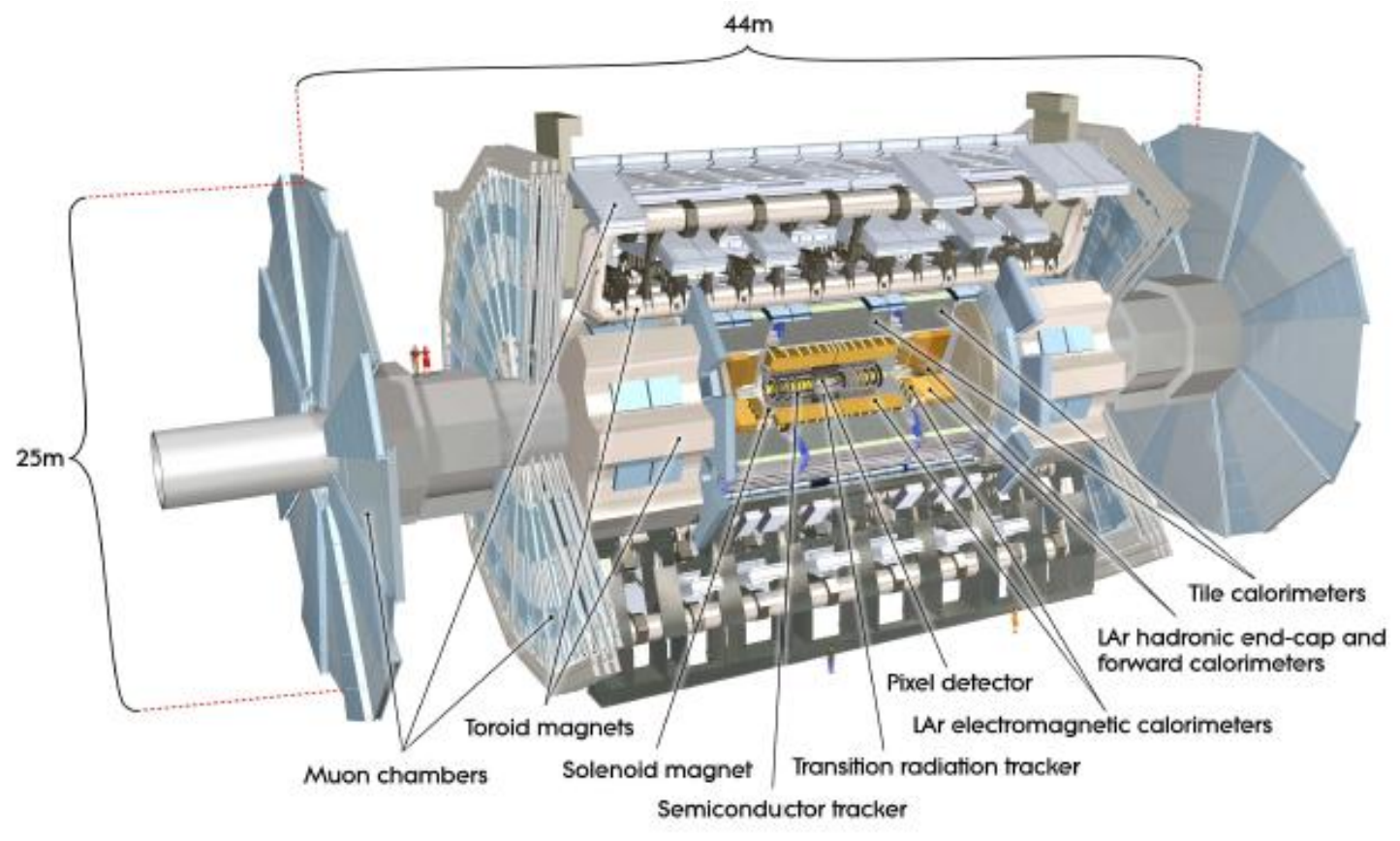

Figure 1: Cutaway view of the ATLAS detector [1]. The dimensions of the detector are $25 \mathrm{~m}$ in height and $44 \mathrm{~m}$ in length. The overall weight of the detector is approximately 7000 tonnes.

The ATLAS detector was constructed by the joint efforts of some 3000 individuals from 174 institutes from 38 countries over the world.

\section{Physics performance}

Thanks to the excellent work of the LHC, the ATLAS experiment has recorded, on top of $5 \mathrm{fb}^{-1}$ collected in 2011 at $7 \mathrm{TeV}$, another $13 \mathrm{fb}^{-1}$ of integrated luminosity at a centre of mass 
energy of $8 \mathrm{TeV}$ up to the opening of this Conference. At the time of writing ATLAS is taking experimental data at a rate of $1 \mathrm{fb}^{-1}$ per week.

Since the start of LHC operations in 2009, data taking efficiency has been higher than $95 \%$ and the fraction of operational channels close to $100 \%$. The detector physics performance is illustrated in Figs. 2 and 3 by the agreement between the data and Monte-Carlo simulation for $J / \Psi$ and $Z$-boson decays. These plots show a good linearity and accuracy of the momentum and energy measurements in the ATLAS detector, from the $Z$ mass scale down to a few $\mathrm{GeV}$.

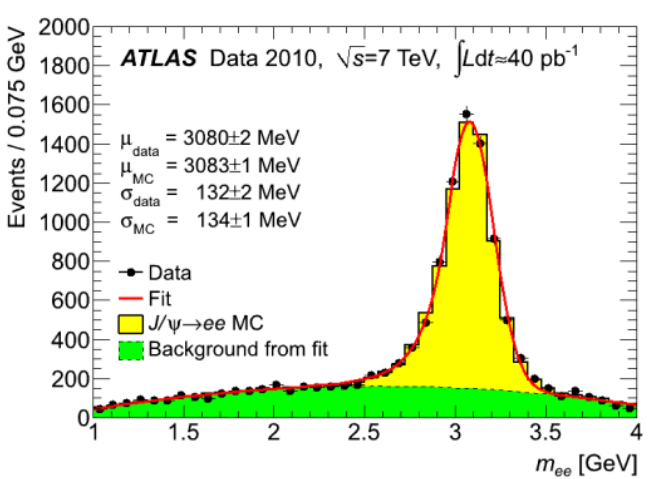

Figure 2: $\quad$ Reconstructed $e^{+} e^{-}$mass distribution in the $J / \Psi$ region for an integrated luminosity of $40 \mathrm{pb}^{-1}$ [2].

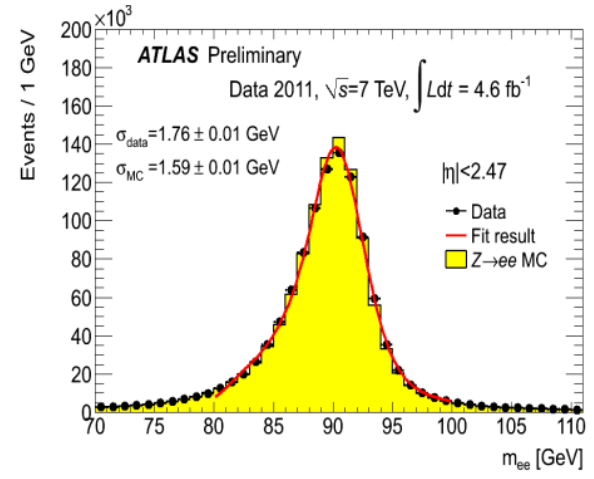

Figure 3: $Z \rightarrow e+e$ - invariant mass for an integrated luminosity of $4.6 \mathrm{fb}^{-1}[2]$.

\section{A few selected results}

A variety of physics results are available from the public page [3] of the ATLAS Collaboration. Only a small number of selected results are presented in this section.

Jet physics is under study in the ATLAS experiment in a new energy regime and unexplored kinematic areas [4]. Fig.4 shows the inclusive jet double-differential cross section as a function of jet transverse momentum, $p_{\mathrm{T}}$, in different regions of $|y|$ for jets identified using the anti- $\mathrm{k}_{\mathrm{t}}$ algorithm with $\mathrm{R}=0.4$ ( $\mathrm{R}$ is defined as a cone size in $\eta-\Phi$ space, where pseudorapidity $\eta$ $=-\ln \tan (\theta / 2), \theta$ is polar angle and $\Phi$ is azimutal angle). For convenience, the cross sections are multiplied by the factors indicated in the legend. The data are compared to perturbative QCD (pQCD) calculations using NLOJET++ to which non-perturbative corrections have been applied [5]. The error bars, which are usually smaller than the symbols, indicate the statistical uncertainty on the measurement. The dark-shaded band indicates the quadratic sum of the experimental systematic uncertainties, dominated by the jet energy scale uncertainty. An overall uncertainty of $3.4 \%$ due to the luminosity measurement is not shown. The theory uncertainty, shown as the light, hatched band, is the quadratic sum of the uncertainties from the choice of the renormalisation and factorisation scales, parton distribution functions, $\alpha_{\mathrm{S}}\left(\mathrm{M}_{\mathrm{Z}}\right)$, and the modelling of non-perturbative effects.

The analysis [4] covers three orders of magnitude of the jet $p_{\mathrm{T}}$, where the inclusive jet cross section value changes by more than six orders. NLO calculations for pQCD were found to be in a good agreement with the experimental data. Good agreement was also observed for the di-jet and multi-jet cross sections [4] as shown in Fig. 5 representing the di-jet doubledifferential cross section as a function of di-jet mass, binned in half the rapidity separation between the two leading jets, $y^{*}=|y 1-y 2| / 2$. 


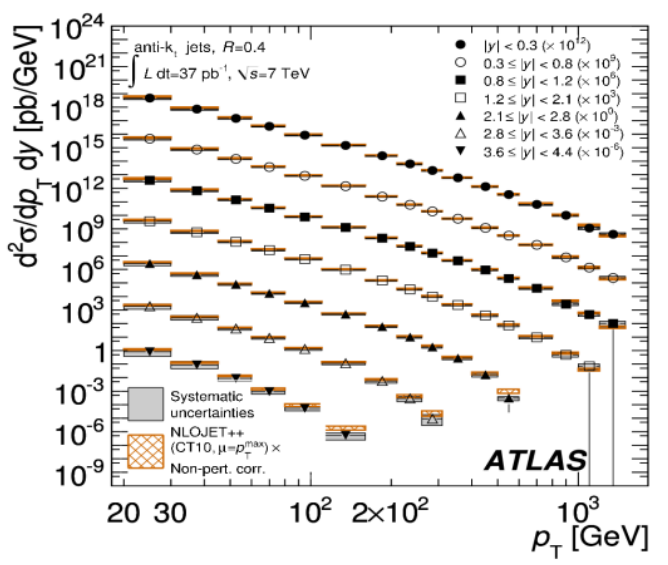

Figure 4: Inclusive jet double-differential cross section as a function of jet $p_{T}$ in different regions of $|y|$. The rapidity $y=1 / 2 \ln \left(\left(E+p_{z}\right) /(E-\right.$ $\left.\left.p_{z}\right)\right)$ is used, where $E$ is the jet energy and $p_{z}$ is the z-component of the jet momentum [4].

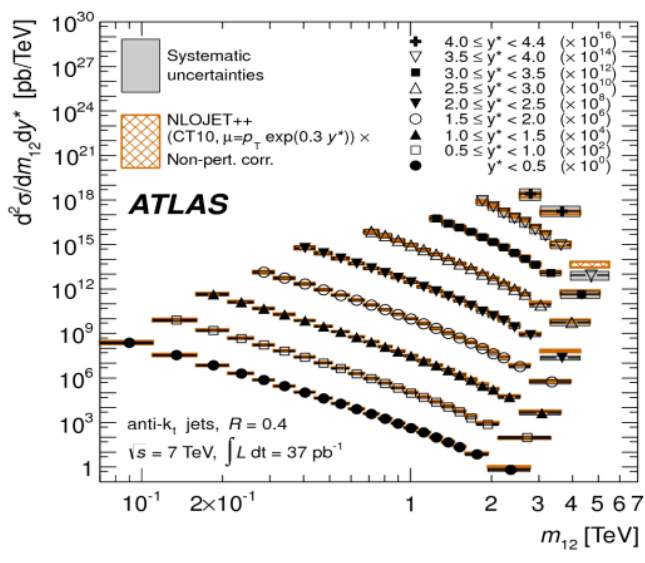

Figure 5: Di-jet double-differential cross section as a function of di-jet mass, binned in half the rapidity separation between the two leading jets, $y^{*}=|y 1-y 2| / 2$ [4].

A summary of electroweak physics in ATLAS is shown in Fig. 6 [3] where the measured cross sections of the main EW processes are presented together with theoretical predictions. The measurements were made with the full set of data from 2010 and 2011, and a part of the 2012 datasets. We are probing the cross sections down to a level of $10 \mathrm{pb}$. The remarkable success of NNLO pQCD calculations together with our present knowledge of the proton parton structure is demonstrated.

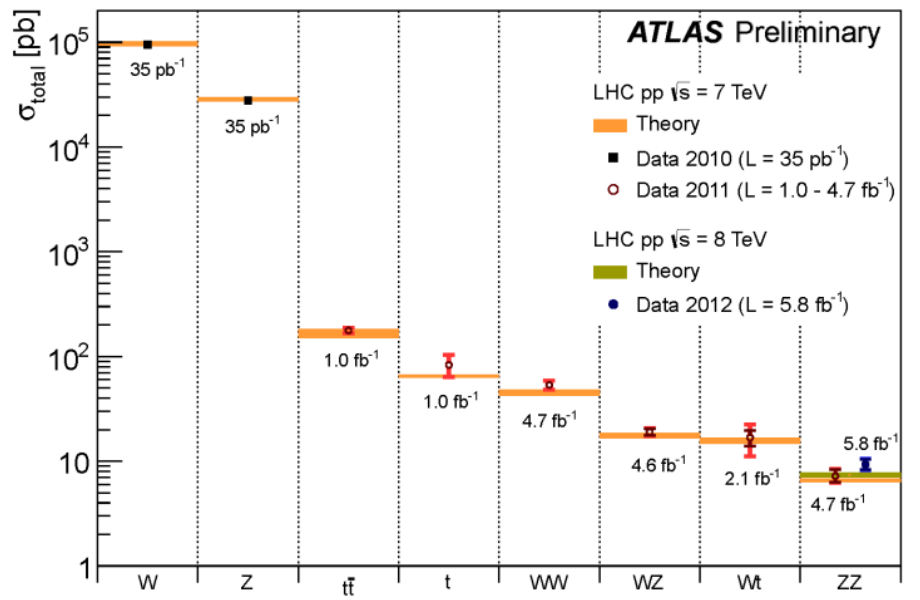

Figure 6: Summary of several Standard Model total production cross section measurements compared to the corresponding theoretical expectations [3]. The dark error bar represents the statistical uncertainly. The red error bar represents the full uncertainty, including systematics and luminosity uncertainties. All theoretical expectations were calculated at NLO or higher.

The good agreement between theoretical calculations and experimental data provides a solid basis for the future discoveries, as the Standard Model proccesses shown in Fig.6 are the backgrounds for Higgs boson production.

Top physics results are presented in Fig.7, which gives a summary of top-pair production cross section measurements [3]. The upper part of the figure shows measurements that are averaged to give the combined value shown. The lower part shows additional newer measurements not included in the combination. The experimental accuracy is already challenging the theoretical predictions indicated by the hatched area. 


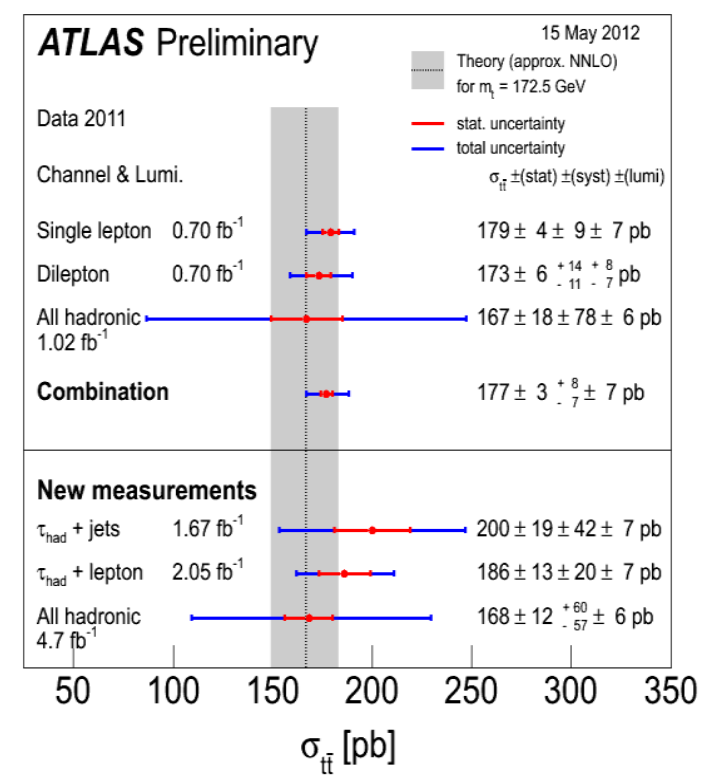

Figure 7: Summary of the measurements of top-pair production cross sections compared to NNLO theoretical calculations [3].

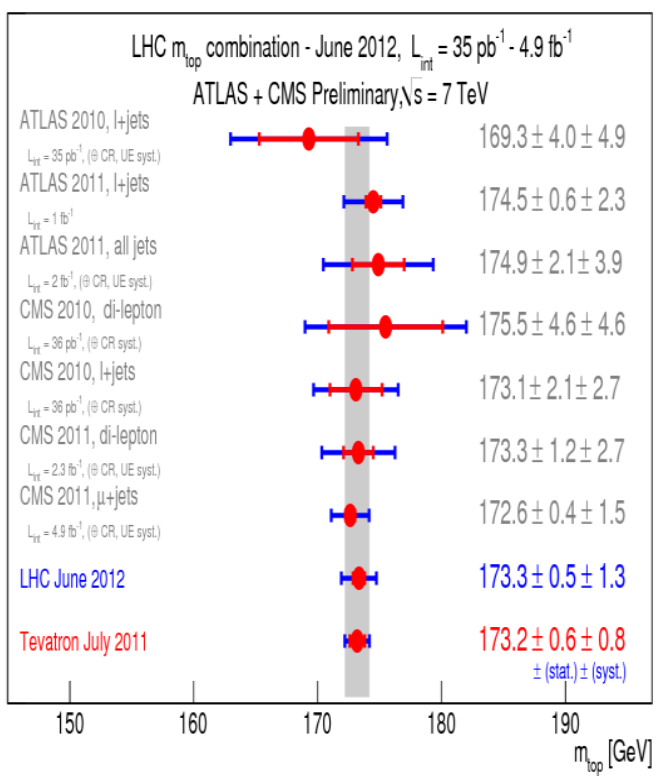

Figure 8: Input measurements and LHC combinations of the top-quark mass measured in ATLAS and CMS experiments at the energy of $7 \mathrm{TeV}$ [3] compared to the Tevatron results and corresponding theoretical expectation.

A combination of the top-quark mass measurements performed by the ATLAS and CMS experiments is presented in Fig.8 [6]. The dataset used corresponds to an integrated luminosity per experiment of up to $4.9 \mathrm{fb}^{-1}$ of proton-proton collisions at a centre-of-mass energy of $7 \mathrm{TeV}$.

The combination includes measurements of the top-antitop decay channels to lepton+jets, to di-lepton and to all-jets channels. The resulting preliminary combined LHC measurement of the top-quark mass is $m_{\text {top }}=173.3 \pm 0.5$ (stat) \pm 1.3 (syst) $\mathrm{GeV}$, with a total uncertainty of $1.4 \mathrm{GeV}$. So far, experiments at the Tevatron have achieved the best accuracy in the top-quark mass measurements and dominate the world average. By the end of 2012 we expect to have an order of magnitude more events than the Tevatron, giving considerably higher precision in our analyses of top-quark properties.

The Heavy Ion program of the ATLAS experiment is aimed at studying the quarkgluon plasma produced in $\mathrm{Pb}+\mathrm{Pb}$ collisions at ${\sqrt{s_{\mathrm{NN}}}}=2.36 \mathrm{TeV}$ centre-of-mass energy of two nucleons. This will be done by studying the pressure-driven collective expansion and the attenuation ("quenches") of high- $p_{\mathrm{T}}$ quarks and gluons not achievable in hadronic systems.

A search for events with final state multiplicity consistent with the initial state gluon production above the critical temperature is also ongoing.

The collective flow of nuclear matter can be described by harmonic flow coefficients [7]. Statistically significant harmonic flow coefficients $v_{\mathrm{n}}(\mathrm{n}>2)$ were observed in ATLAS. They are shown in Fig. 9 for different values of particle $p_{\mathrm{T}}$ and event centrality: $v_{3}>v_{2}$ is seen in very central $(0-5 \%) \mathrm{Pb}+\mathrm{Pb}$ collisions. 


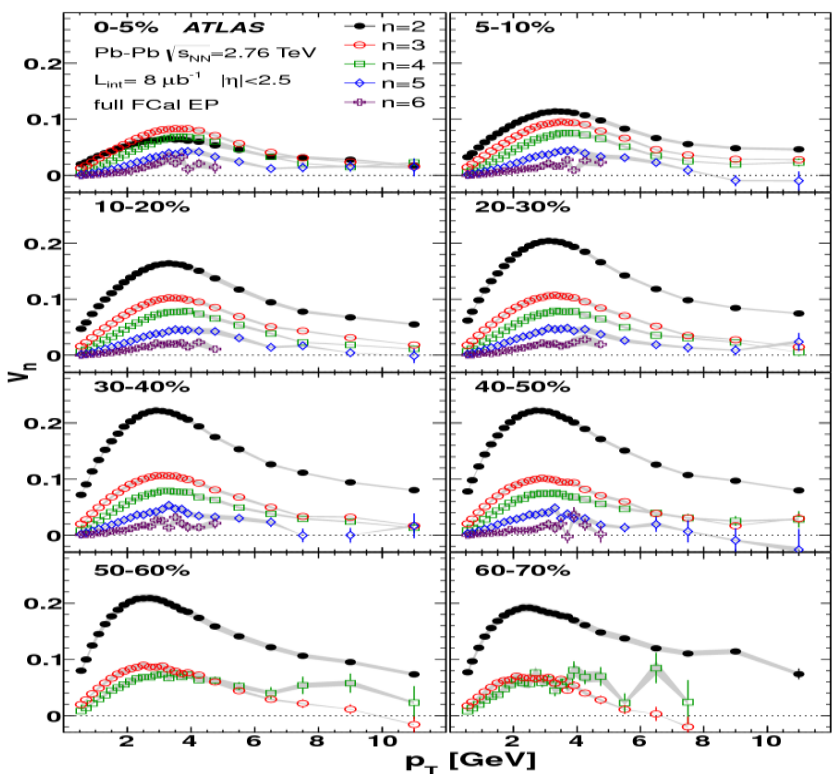

At present ATLAS is focusing on using high- $p_{\mathrm{T}}$ jets, di-jets and multi-jet final states, as well as quarkonia $-Y(1 \mathrm{~S}), Y(2 \mathrm{~S})$ and $Y(3 \mathrm{~S})$ states - to study Debye screening. The cold nuclear matter effects and low- $x$ processes will also be studied in $\mathrm{p}+\mathrm{Pb}$ collisions.

Figure 9: $\quad v_{\mathrm{n}}$ vs $p_{\mathrm{T}}$ for several centrality intervals. The shaded bands indicate the systematic uncertainties [7].

\section{Observation of a new Higgs boson-like particle}

A successful climax to the search for the Higgs boson was demonstrated in the presentation of the ATLAS and the CMS results at the ICHEP-2012 conference in Melbourne. The observation of a new particle has opened a new chapter in a history of particle physics and is a great achievement of both the ATLAS and CMS experiments [8,9]. Various production channels were combined to obtain higher significance for observation of the Standard Model Higgs boson.

The table below [8] summarises the individual channels entering the combination. The transition points between separately optimized $m_{\mathrm{H}}$ regions are indicated. In channels sensitive to associated production of the Higgs boson, $V$ indicates a $W$ or $Z$ boson. The symbols $\otimes$ and $\oplus$ represent direct products and sums over sets of selection requirements, respectively.

\begin{tabular}{|c|c|c|c|c|}
\hline $\begin{array}{c}\text { Higgs Boson } \\
\text { Decay }\end{array}$ & $\begin{array}{c}\text { Subsequent } \\
\text { Decay }\end{array}$ & Sub-Channels & $\begin{array}{c}m_{H} \text { Range } \\
{[\mathrm{GeV}]}\end{array}$ & $\begin{array}{l}\int \mathrm{L} d t \\
{\left[\mathrm{fb}^{-1}\right]}\end{array}$ \\
\hline \multicolumn{5}{|c|}{$2011 \sqrt{s}=7 \mathrm{TeV}$} \\
\hline \multirow{3}{*}{$H \rightarrow Z Z^{(*)}$} & $4 \ell$ & $\{4 e, 2 e 2 \mu, 2 \mu 2 e, 4 \mu\}$ & $110-600$ & 4.8 \\
\hline & $\ell \ell v \bar{v}$ & $\{e e, \mu \mu\} \otimes\{$ low, high pile-up $\}$ & $200-280-600$ & 4.7 \\
\hline & $\ell \ell q \bar{q}$ & $\{b$-tagged, untagged $\}$ & $200-300-600$ & 4.7 \\
\hline$H \rightarrow \gamma \gamma$ & - & 10 categories $\left\{p_{\mathrm{Tt}} \otimes \eta_{\gamma} \otimes\right.$ conversion $\} \oplus\{2$-jet $\}$ & $110-150$ & 4.8 \\
\hline \multirow{2}{*}{$H \rightarrow W W^{(*)}$} & $\ell v \ell v$ & $\{e e, e \mu / \mu e, \mu \mu\} \otimes\{0$-jet, 1-jet, 2-jet $\} \otimes\{$ low, high pile-up $\}$ & $110-200-300-600$ & 4.7 \\
\hline & $\ell v q q^{\prime}$ & $\{e, \mu\} \otimes\{0$-jet, 1-jet, 2-jet $\}$ & $300-600$ & 4.7 \\
\hline \multirow{3}{*}{$H \rightarrow \tau \tau$} & $\tau_{\text {lep }} \tau_{\text {lep }}$ & $\{e \mu\} \otimes\{0$-jet $\} \oplus\{\ell \ell\} \otimes\{1$-jet, 2-jet, $V H\}$ & $110-150$ & 4.7 \\
\hline & $\tau_{\text {lep }} \tau_{\text {had }}$ & $\begin{array}{c}\{e, \mu\} \otimes\{0 \text {-jet }\} \otimes\left\{E_{\mathrm{T}}^{\text {miss }}<20 \mathrm{GeV}, E_{\mathrm{T}}^{\text {miss }} \geq 20 \mathrm{GeV}\right\} \\
\oplus\{e, \mu\} \otimes\{1 \text {-jet }\} \oplus\{\ell\} \otimes\{2 \text {-jet }\}\end{array}$ & $110-150$ & 4.7 \\
\hline & $\tau_{\text {had }} \tau_{\text {had }}$ & $\{1$-jet $\}$ & $110-150$ & 4.7 \\
\hline \multirow{3}{*}{$V H \rightarrow V b b$} & $Z \rightarrow v v$ & $E_{\mathrm{T}}^{\mathrm{miss}} \in\{120-160,160-200, \geq 200 \mathrm{GeV}\}$ & $110-130$ & 4.6 \\
\hline & $W \rightarrow \ell v$ & $p_{\mathrm{T}}^{W^{1}} \in\{<50,50-100,100-200, \geq 200 \mathrm{GeV}\}$ & $110-130$ & 4.7 \\
\hline & $Z \rightarrow \ell \ell$ & $p_{\mathrm{T}}^{Z} \in\{<50,50-100,100-200, \geq 200 \mathrm{GeV}\}$ & $110-130$ & 4.7 \\
\hline \multicolumn{5}{|c|}{$2012 \sqrt{s}=8 \mathrm{TeV}$} \\
\hline$H \rightarrow Z Z^{(*)}$ & $\overline{4 \ell \ell}$ & $\{4 e, 2 e 2 \mu, 2 \mu 2 e, 4 \mu\}$ & $110-600$ & 5.8 \\
\hline$H \rightarrow \gamma \gamma$ & - & 10 categories $\left\{p_{\mathrm{Tt}} \otimes \eta_{\gamma} \otimes\right.$ conversion $\} \oplus\{2$-jet $\}$ & $110-150$ & 5.9 \\
\hline$H \rightarrow W W^{(*)}$ & $e v \mu v$ & $\{e \mu, \mu e\} \otimes\{0$-jet, 1-jet, 2-jet $\}$ & $110-200$ & 5.8 \\
\hline
\end{tabular}

An excess of events was observed near mass $m_{\mathrm{H}}=126 \mathrm{GeV}$ in the most sensitive channels $H \rightarrow Z Z^{(*)} \rightarrow 4 \ell$ and $H \rightarrow \gamma \gamma$, both providing fully reconstructed candidates with high 
resolution in invariant mass. The statistical procedure to interpret the data is described in Ref. [8] and references therein. The significance of an excess in the data is quantified by the local $p_{0}$, the probability that the background can produce a fluctuation greater than or equal to the excess observed in data. The observed local $p_{0}$ values from the combination of channels are shown as a function of $m_{\mathrm{H}}$ in Fig. 10 for the low mass range. The mass of the observed new particle was estimated for the channels with highest mass resolution as $126.0 \pm 0.4$ (stat) \pm 0.4 (sys) GeV

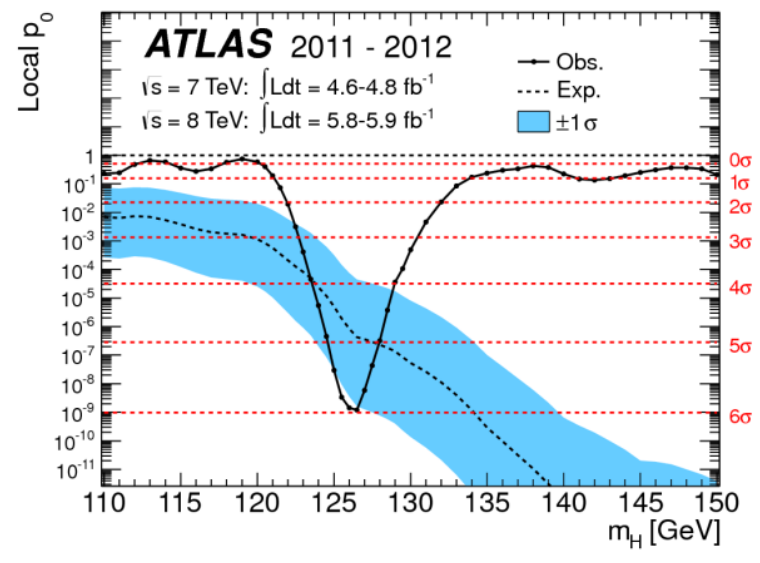

Figure 10: The observed (solid) local $p_{0}$ as a function of $m_{H}$ in the low mass range. The dashed curve shows the expected local $p_{0}$ under the hypothesis of a SM Higgs boson signal at that mass with its $\pm 1 \sigma$ band. The horizontal dashed lines indicate the p-values corresponding to significances of 1 to $6 \sigma[8]$.

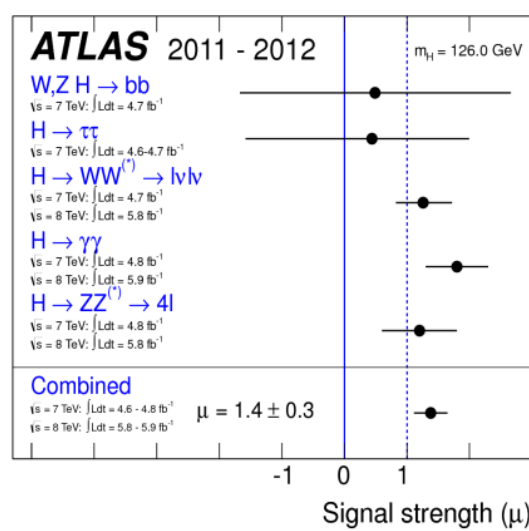

Figure 11: Measurements of the signal strength parameter $\mu$ for $m_{H}=126 \mathrm{GeV}$ for the individual channels and their combination [8].

The parameter of interest is the global signal strength factor $\mu$, which acts as a scale factor on the total number of SM Higgs events. The value $\mu=0$ corresponds the background-only hypothesis, and $\mu=1$ to SM Higgs in addition to background. The hypothesized values of signal strength were tested using the profile likelihood ratio [10]. A summary of the individual and combined best-fit values of the strength parameter for a SM Higgs boson mass hypothesis of $126 \mathrm{GeV}$ is shown in Fig. 11. It demonstrates consistency of the observation with the SM expectation.

\section{ATLAS upgrade}

The LHC experiments are following the collider upgrade program in order to enhance their discovery potentials and search sensitivities. The ATLAS experiment is interested in a luminosity upgrade (HL-LHC) of the LHC to an instantaneous luminosity up to $5 \cdot 10^{34} \mathrm{~cm}^{-2} \mathrm{~s}^{-1}$. The higher statistics will provide the opportunities for precision measurements of the mass of the Higgs boson candidate, for setting an upper limit on its width, and for measurements of its couplings to $W, Z$ and fermions. The proposed integrated luminosity of $300 \mathrm{fb}^{-1}$ at $13 \mathrm{TeV}$ will allow an accurate measurements of the couplings and study of rare decays such as $H \rightarrow \mu \mu$ and $H \rightarrow Z \gamma$ and $V V$ scattering $(V=W, Z)$.

The published Letter of Intent [11] presents the ATLAS Collaboration's plans for a detector upgrade. The main detector sub-systems addressed in these plans are:

- Muon system: a new tracking and trigger device to be installed in the inner layer of 
the forward spectrometer, that will not only provide a sharper trigger threshold but also greatly improve the tracking performance under the higher backgrounds expected at HL-LHC;

- Calorimeters: new trigger read-out boards will be implemented to exploit the longitudinal sampling of the calorimeter and higher trigger granularity, which will lead to an improvement in rejecting fake electron triggers;

- AFP: construction of additional ATLAS Forward Proton detectors at distances of $\sim 200 \mathrm{~m}$ from the interaction point;

- $\quad$ FTK - Fast Tracking trigger: a pipeline electronics system for the fast track processing in Si-detectors at the first level of the three-level trigger;

- Trigger/DAQ upgrades, etc.

The plans are clearly defined for the so called Phase I of LHC upgrade for the period up to 2018; the preparation for the Phase II (2022) is ongoing.

\section{Conclusions}

The ATLAS experiment has been performing well in 2012. The Standard Model Higgs boson is excluded at $95 \%$ Confidence Level in the mass range $111-559 \mathrm{GeV}$, except for the narrow region $122-131 \mathrm{GeV}$. In this region, an excess of events with significance $5.9 \sigma$ is observed. Many interesting physics analyses have been reported at the summer conferences and are presented in the ATLAS Collaboration public page. The strategy of the future analyses and detector development is shaping up to provide even better opportunities for expected new discoveries.

\section{References}

[1] ATLAS Collaboration, JINST 3 S08003 (2008)

[2] ATLAS Collaboration, CERN-PH-EP-2011-117 (2011); arXiv:1110.3174 [hep-ex]

[3] https://twiki.cern.ch/twiki/pub/AtlasPublic/

[4] ATLAS Collaboration, Phys.Rev. D86 (2012) 014022

[5] ATLAS Collaboration, ATLAS-CONF-2010-031 (2010); https://cdsweb.cern.ch/record/1277665

[6] ATLAS Collaboration, ATLAS-CONF-2012-095 (2012); https://cdsweb.cern.ch/record/1460441

[7] ATLAS Collaboration, Phys.Rev. C86 (2012) 014907

[8] ATLAS Collaboration, Phys. Lett. B716 (2012) 1-29

[9] CMS Collaboration, Phys. Lett. B716 (2012) .

[10] G.Cowan et al., Eur. Phys. J. C71 (2011) 1554

[11] ATLAS Collaboration, CERN-LHCC-2011-012 